\title{
Correction to: Electrochemical-Based Surface Enhancement of Additively Manufactured Ti-6Al-4V Complex Structures
}

Haniyeh Fayazfar, Issa Rishmawi, and Mihaela Vlasea

Published online: 9 August 2021

\section{Correction to:}

\section{JMEPEG (2021) 30:2245-2255 \\ https://doi.org/10.1007/s11665-021-05512-x}

In Fig. 5(a), images for electropolishing times of 250 and $700 \mathrm{~s}$ were labeled incorrectly. The images in fact correspond to electropolishing times of 150 and $250 \mathrm{~s}$, respectively. In Fig. 7(a), SEM images of as-built and polished surfaces were mislabeled. The image labeled as the as-built rectangular sample shows the as-built U-shaped sample. The image labeled as the polished cylindrical sample shows the polished rectangular sample, and the image labeled as the polished U-shaped sample represents the polished rectangular sample.

The images have been re-taken using a single SEM instrument to ensure consistency across samples, and each image has been labeled correctly.

The original articles can be found online at https://doi.org/10.1007/ s11665-021-05512-x.

Haniyeh Fayazfar, Department of Mechanical and Mechatronics Engineering, University of Waterloo, Waterloo, ON N2L 3G1, Canada; and Department of Mechanical and Manufacturing Engineering, University of Ontario Tech, Oshawa, ON L1G 0C5, Canada; and Issa Rishmawi and Mihaela Vlasea, Department of Mechanical and Mechatronics Engineering, University of Waterloo, Waterloo, ON N2L 3G1, Canada. Contact e-mail: ramona.fayazfar@ontariotechu.ca. 

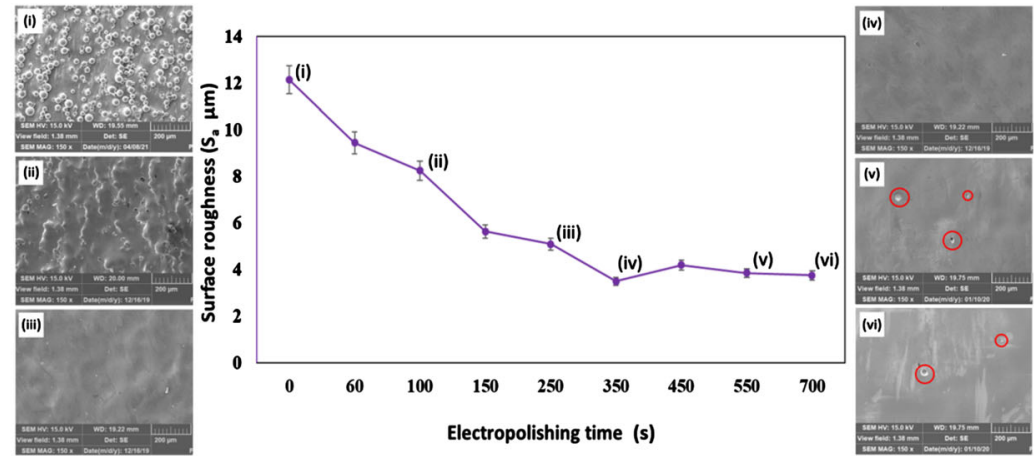

(a)
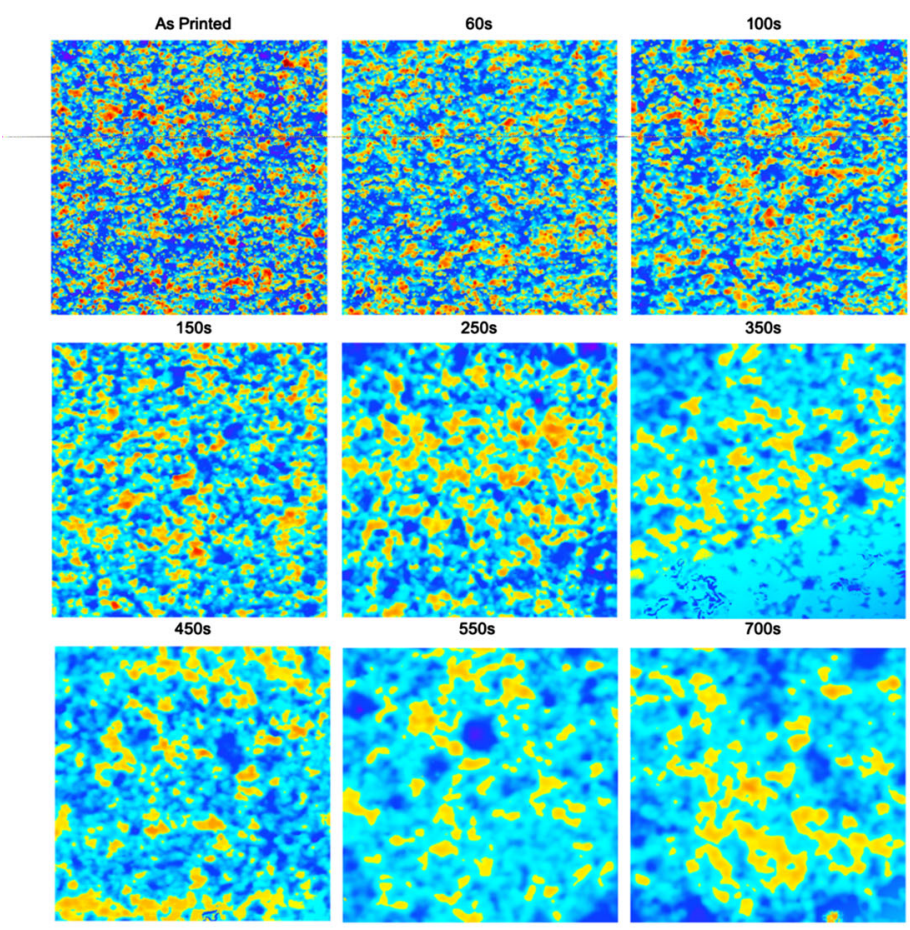

(b)
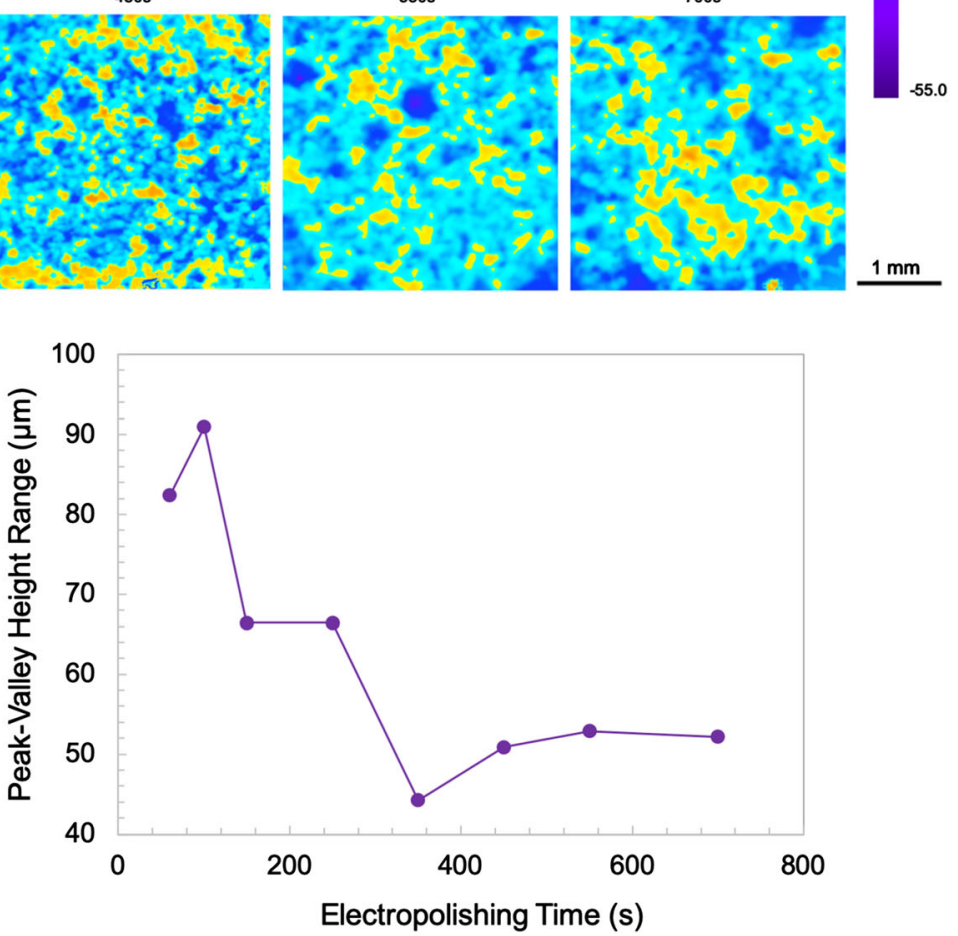

Fig. 5 Surface characterization on the rectangular bar samples; (a) SEM (magnification X100) images of the surface of the as-built and electropolished samples (all SEM scales are the same for all images); (b) surface profiles with various EP time; and (c) peak-valley height range for different EP time 
As-built
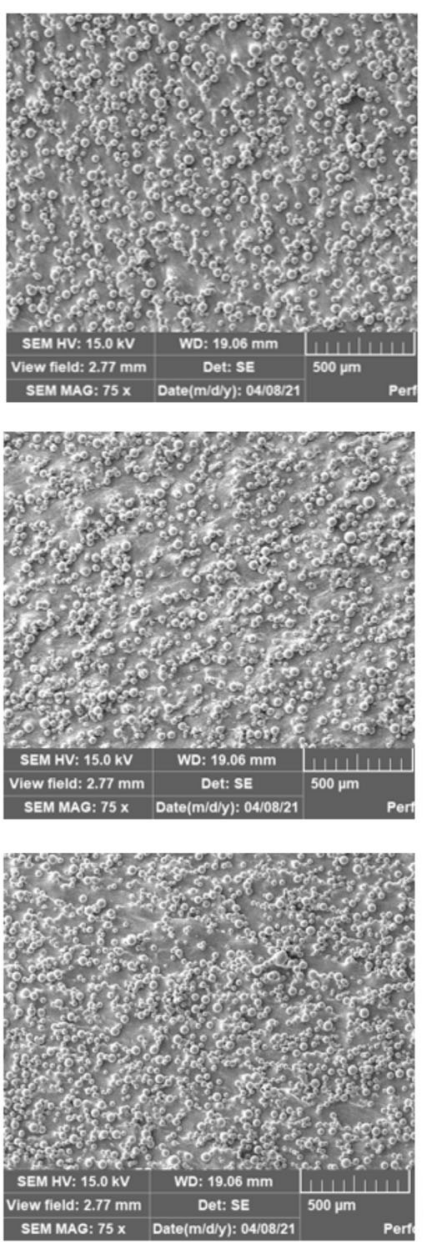

(a)
Polished
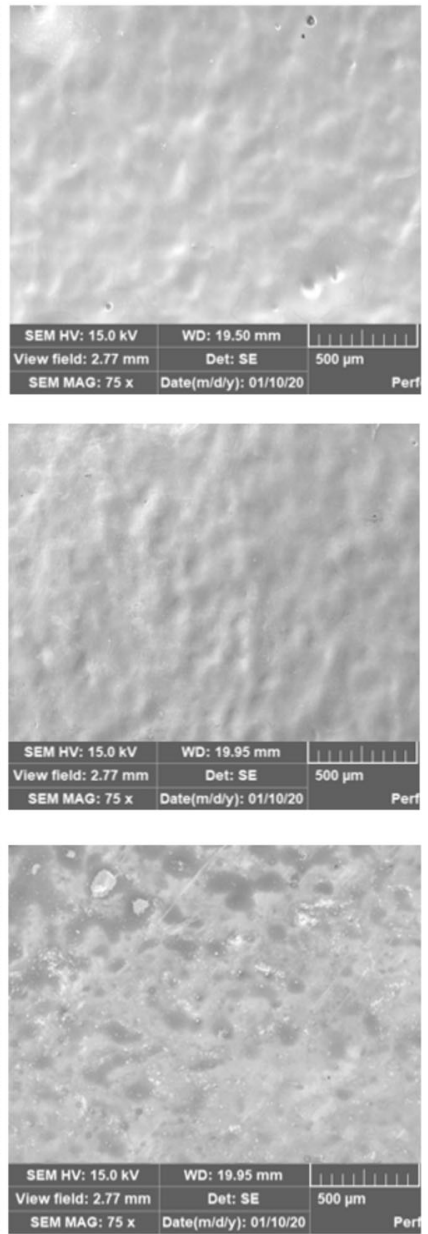

As-built
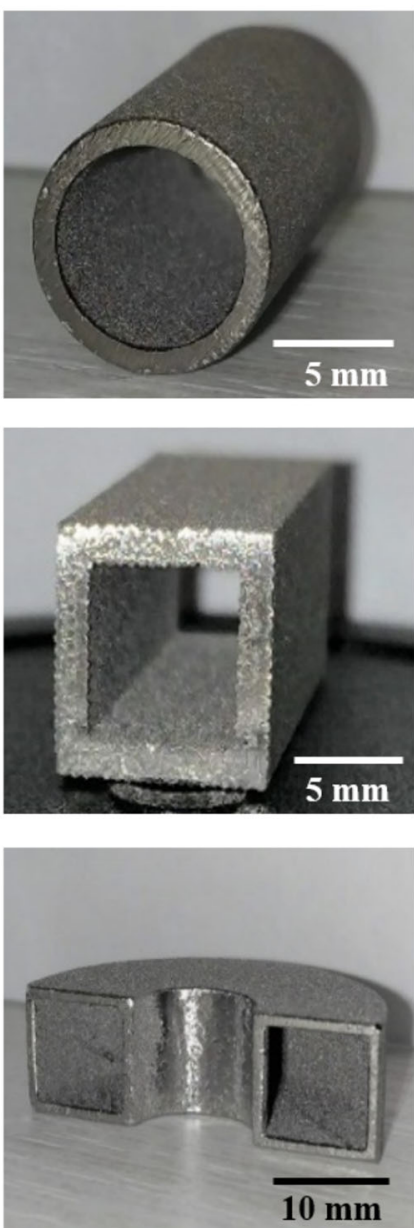

(b)
Polished
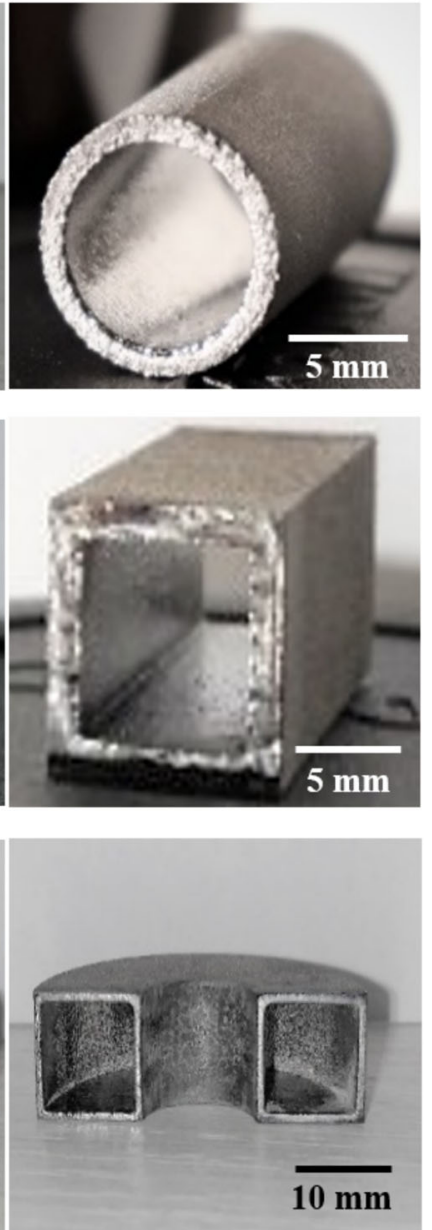

Fig. 7 (a) SEM micrographs of as-built (left column) and electropolished (right column) samples with various geometries, (b) optical images of as-built (left column) and electropolished (right column) samples; the surfaces here were polished for $350 \mathrm{~s}$ except U-shape part which was polished for $800 \mathrm{~s}$

\section{Acknowledgments}

The authors wish to acknowledge Dr. Hamed Asgari for assistance with SEM imaging.
Publisher's Note Springer Nature remains neutral with regard to jurisdictional claims in published maps and institutional affiliations. 